\title{
Preservation of rutin nanosuspensions without the use of preservatives
}

\author{
Pascal L. Stahr and Cornelia M. Keck ${ }^{*} \S$
}

Full Research Paper

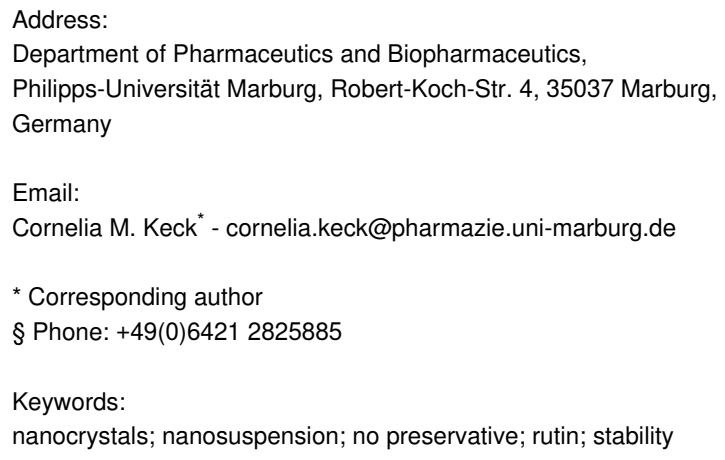

Beilstein J. Nanotechnol. 2019, 10, 1902-1913. doi:10.3762/bjnano.10.185

Received: 09 April 2019

Accepted: 27 August 2019

Published: 19 September 2019

This article is part of the thematic issue "Frontiers in pharmaceutical nanotechnology".

Guest Editor: M. G. Wacker

(c) 2019 Stahr and Keck; licensee Beilstein-Institut. License and terms: see end of document.

\begin{abstract}
Nanocrystals are used as universal approach to improve the bioactivity of poorly soluble active ingredients. They are produced by various techniques, typically yielding aqueous nanosuspensions, which are prone to microbial contamination. Preservation of nanocrystals is possible but might not always be feasible, as preservatives might interfere with other excipients in the formulations or with chemicals used in assays, cell cultures or animal models. Therefore, to enable an easier use of nanocrystals, preservative-free nanosuspensions would be a good alternative. In this study, rutin nanocrystals were frozen and stored for three months at $-20{ }^{\circ} \mathrm{C}$. The chemical, physical and microbial stability were monitored, and the results were compared to preserved nanosuspensions. The frozen nanosuspensions remained stable and possessed excellent stability over the whole time of storage, indicating that the freeze-thaw process is suitable for the production of preservative-free nanosuspensions with excellent long-term stability. The freeze-thaw process for nanosuspensions is a simple concept and is suggested as alternative, when preserved nanosuspensions cannot be used.
\end{abstract}

\section{Introduction}

Nanocrystals for pharmaceutical use were invented in the early 1990s [1-4]. They are composed of $100 \%$ substance, are stabilized with only small amounts of surfactants, and possess particle sizes below $1 \mu \mathrm{m}$. According to the Ostwald-Freundlich equation nanocrystals possess a higher curvature leading to an enhanced dissolution pressure and thus to an enhanced kinetic saturation solubility [5]. Due to their small size they possess an

increased surface area, resulting in an increased dissolution rate expressed by the Noyes-Whitney equation. In addition, they also possess an increased adhesiveness and thus, represent a universal, powerful and well-known formulation principle to overcome poor aqueous solubility and poor bioavailability of class-II and class-VI active ingredients of the biopharmaceutics classification system (BCS) [6,7]. Nanocrystals are already used 
in various pharmaceutical drug products for oral use. Examples are Rapamune ${ }^{\circledR}$ (Sirolimus, Wyeth), Emend ${ }^{\circledR}$ (Aprepitant, Merck), Tricor ${ }^{\circledR}$ (Fenofibrate, Abbott), Megace ES ${ }^{\circledR}$ (Megestrol, Par Pharm) or Triglide ${ }^{\circledR}$ (First Horizon Pharmaceuticals). In 2009 the first parenteral drug product, Invega Sustenna ${ }^{\circledR}$ (Paliperidone palmitate, Johnson \& Johnson), was approved by the FDA. However, besides oral or parenteral administration, nanocrystals can also be used to improve the bioactivity of poorly soluble active ingredients via other routes of administration. Examples include pulmonal, ocular or dermal application [8-12].

Nanocrystals can be produced by different methods. Examples are precipitation, wet milling, high-pressure homogenization or combinations of these methods [1-4]. Regardless of the process used, all these methods will yield nanosuspensions, i.e., nanocrystals dispersed in a liquid. As liquid formulations are not always a convenient dosage form for the final drug product, in most cases nanosuspensions need to be formulated into other, more convenient, dosage forms. Depending on the route of administration this could be tablets, pellets, powders, gels or creams. However, prior to the formulation into final drug products, the aqueous nanosuspensions need to be stored, which certainly requires a sufficient stability of the nanosuspension. For this, besides chemical and physical stability, also the microbial stability needs to be considered.

One method to avoid microbial contamination of aqueous formulations during storage is the use of preservatives. In previous studies it was already found that preservatives can strongly impair the physical stability of the nanosuspensions. Reasons for this are changes of $\mathrm{pH}$ value or of the conductivity of the dispersion medium, or the adsorption of the preservatives onto the surface of the particles, which changes the charge of the particles (zeta potential) and forces agglomeration of the nanocrystals. To avoid instabilities of nanosuspensions only very hydrophilic and non-charged preservatives, which will not interact with the nanocrystals, should be used. Due to the above-mentioned reasons, only a few preservatives are available for the preservation of nanocrystals. Suitable preservatives for the preservation of nanocrystals include different alcohols, i.e., pentylene glycol or mixtures of phenoxyethanol and ethyl hexyl glycerol [13-15].

The limited number of preservatives and sometimes the incompatibility of these preservatives with other excipients in the final formulation are inconvenient for a successful formulation of nanosuspensions. Therefore, to enable a more convenient formulation of nanocrystals in the future, this work aimed at investigating an alternative method to maintain the microbial stability of nanosuspensions during storage.
Considering that bacterial growth strongly depends on the temperature, it was hypothesized that freezing of non-preserved nanosuspensions might prevent bacterial growth of the nanosuspensions during storage. However, the harsh conditions during freezing and thawing might also impair the physical stability of the nanocrystals and might cause agglomeration of the particles, which would then lead to a loss of the "nano properties". Hence, in this case the method could not be exploited to preserve nanosuspensions without preservative.

To investigate whether the freeze-thaw method is suitable for the production of long-term stable non-preserved nanosuspensions with high microbial quality, previously developed nanosuspensions containing the flavonoid rutin as model substance and either Plantacare 2000 or Poloxamer 188 (PLX 188) as stabilizers were produced by high-pressure homogenization as described previously [16-19]. Each of the nanosuspensions obtained was allocated into two parts. One part was preserved, and the other part remained non-preserved. All formulations were stored at different temperatures for a period of three months and size, zeta potential, antioxidant capacity and the microbial quality were determined and monitored over this period of time (Figure 1).

\section{Results and Discussion Production and characterization of nanosuspensions}

High-pressure homogenization yielded rutin nanosuspensions with a relatively broad size distribution, i.e., polydispersity indices (PdI values) above 0.3 and some larger particles with sizes above $4 \mu \mathrm{m}$ (Table 1). Because of this, the nanosuspensions were expected to be prone to Ostwald ripening, i.e., particle growth during storage was expected. Limited physical stability of suspensions is advantageous if a study aims at investigating different stabilizing and destabilizing effects, because in comparison to highly physically stable formulations, destabilizing effects can be detected earlier during storage, making a discrimination between stabilizing and destabilizing effects clearer.

The suspension stabilized with PLX 188 yielded sizes of about $410 \mathrm{~nm}$. Slightly larger nanocrystals with a slight agglomeration were obtained when Plantacare was used as stabilizer (Table 1 and Table 2). From this it was expected that nonpreserved nanosuspensions stabilized with PLX 188 might possess a slightly better physical stability than the Plantacare-stabilized formulations. Upon the addition of the preservatives only very minor changes in size were observed for both formulations (Table 1) and for the Plantacare-stabilized formulation even a slight deagglomeration was determined (Table 2). Also the zeta potential values did not change significantly in both 


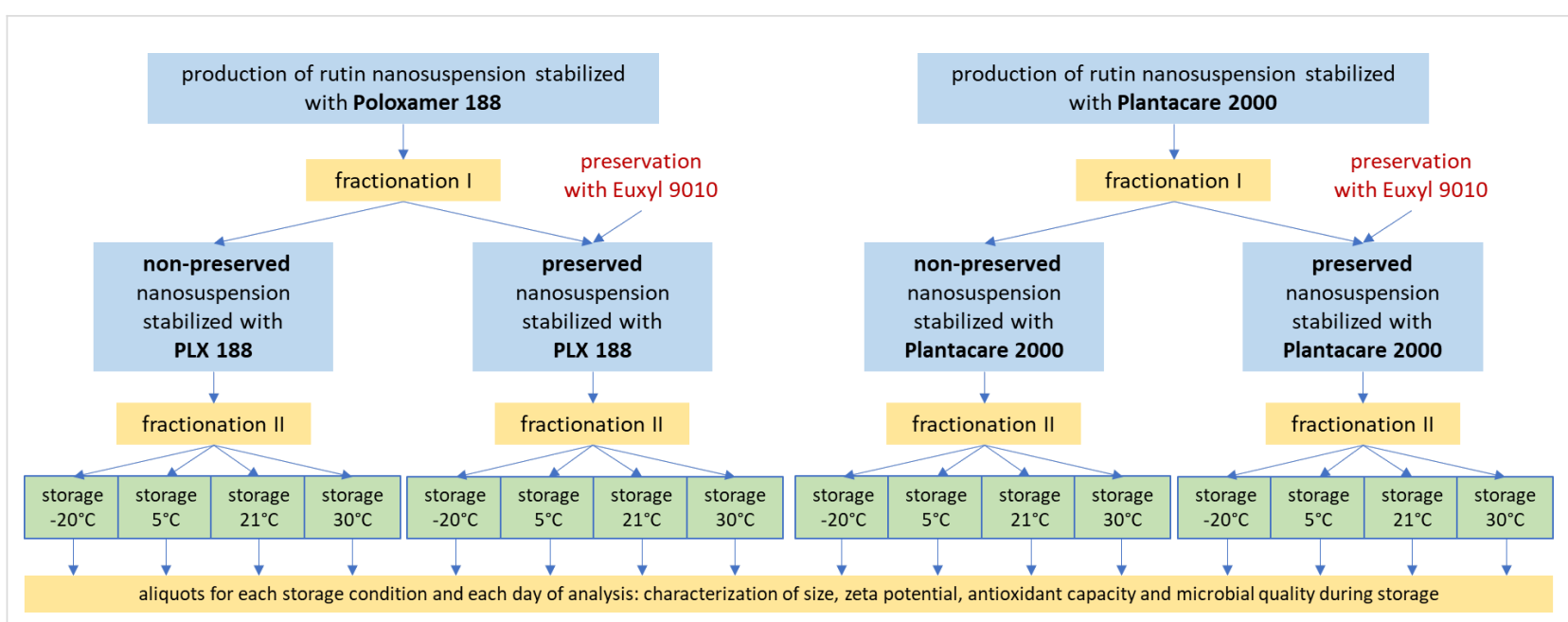

Figure 1: Scheme of the study. Rutin nanosuspensions with two different stabilizers were produced. The formulations were allocated, one aliquot was preserved, and the other part remained non-preserved. All formulations obtained were stored at different temperatures for three months. During this time changes in size, zeta potential, antioxidant capacity and microbial quality were monitored.

\begin{tabular}{|c|c|c|c|c|c|c|c|}
\hline \multirow[t]{2}{*}{ stabilizer } & \multirow[t]{2}{*}{ preservative } & \multicolumn{2}{|c|}{ DLS data } & \multicolumn{2}{|c|}{ zeta potential [mV] } & \multicolumn{2}{|c|}{ LD data $[\mu \mathrm{m}]^{\mathrm{a}}$} \\
\hline & & $\mathrm{z}$-ave $[\mathrm{nm}]$ & $\mathrm{Pdl}$ & in water & in medium & $d(v) 0.50$ & $d(v) 0.95$ \\
\hline \multirow[t]{2}{*}{ PLX 188} & no preservative & $408 \pm 45$ & $0.31 \pm 0.08$ & $-29.4 \pm 2.9$ & $-24.8 \pm 2.6$ & $1.3 \pm 0.17$ & $4.09 \pm 0.15$ \\
\hline & with preservative & $412 \pm 29$ & $0.30 \pm 0.05$ & $-27.0 \pm 7.8$ & $-24.6 \pm 3.0$ & $1.3 \pm 0.12$ & $4.13 \pm 0.15$ \\
\hline \multirow[t]{2}{*}{ Plantacare 2000} & no preservative & $436 \pm 30$ & $0.32 \pm 0.06$ & $-30.6 \pm 4.0$ & $-39.5 \pm 2.7$ & $1.2 \pm 0.11$ & $4.02 \pm 0.11$ \\
\hline & with preservative & $447 \pm 15$ & $0.33 \pm 0.06$ & $-29.3 \pm 2.7$ & $-37.6 \pm 3.3$ & $1.2 \pm 0.12$ & $3.99 \pm 0.11$ \\
\hline
\end{tabular}

${ }^{a} d(v)$ : volumetric median diameter.

water and original dispersion medium (Table 1), again indicating no, or only a very limited, impairment of the stabilization mechanisms of the nanocrystals by the hydrophilic preservative [20].

\section{Physical stability}

The physical stability was assessed by size measurements over a period of three months of samples under all storage conditions. Increases in $d(v)$ values, $z$-average, and polydispersity index (PdI) over time indicated instability. The laser diffractometry (LD) data and the dynamic light scattering (DLS) data obtained from this part of the study are shown in Figures $2-7$.

For the non-preserved nanosuspensions, increasing storage temperatures reduced the physical stability of the non-preserved suspensions (Figure 2, Figure 3, Figure 6, Figure 7). Consequently, the least stable formulations were obtained when the formulations were stored at $30{ }^{\circ} \mathrm{C}$. Reasons for this are the more pronounced particle growth due to Ostwald ripening at elevated temperatures and/or destabilization due to accelerated bacterial growth, which might excrete compounds that contribute to changes in $\mathrm{pH}$ value or conductivity in the medium or interact with the particles. Such changes should become visible in the zeta potential values. However, this was not the case in this study (Figure 8). Therefore, the observed destabilization at elevated temperatures of the non-preserved nanosuspensions might be more related to Ostwald ripening. The assumption is also underlined by the fact that the Poloxamer-stabilized formulations, which possessed a narrower size distribution, i.e., no agglomerates at the day of production (c.f. Table 2), were found to be more stable than the Plantacare-stabilized formulations with a broader size distribution due to a slight agglomeration of the nanocrystals at the day of production.

The trend for physical stability was different for the preserved nanosuspensions. The most stable formulations were obtained when the samples were stored at room temperature. Lower (except freezing) and higher temperatures reduced the stability (Figures 4-7). Reasons for this cannot be explained completely but might be due to the presence of the preservatives that were added to the samples at room temperature. Changes in tempera- 
Table 2: Microscopic images of the nanosuspensions stabilized with Poloxamer 188 (left) and Plantacare 2000 (right) at the day of production. Magnification: 400 -fold.

\section{stabilized with Poloxamer 188}

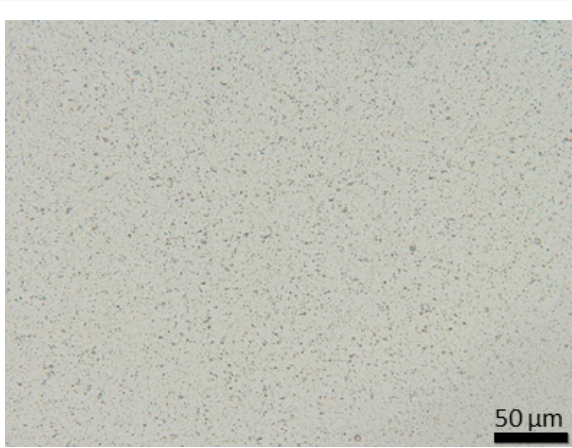

preserved stabilized with Plantacare 2000

non-preserved

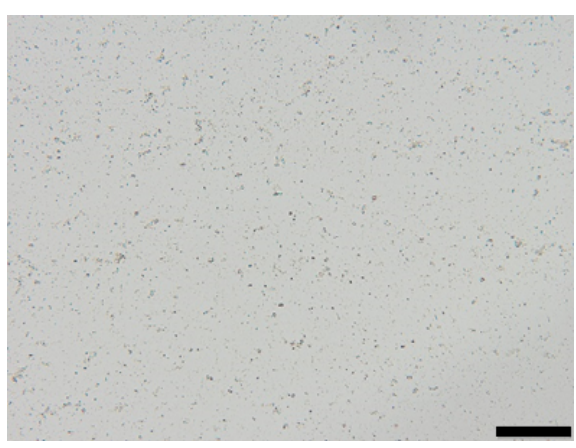

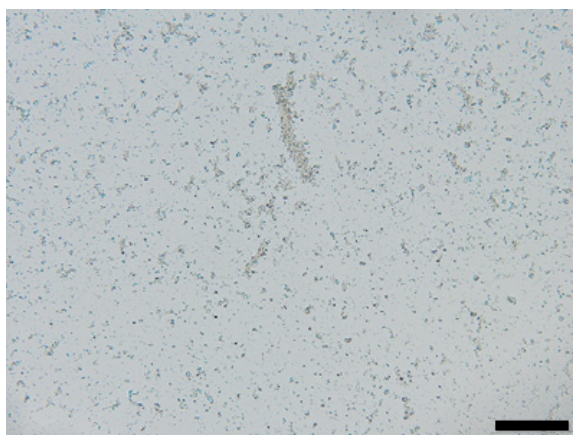

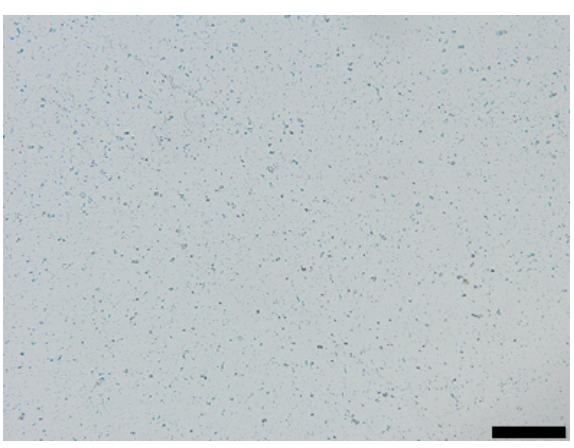

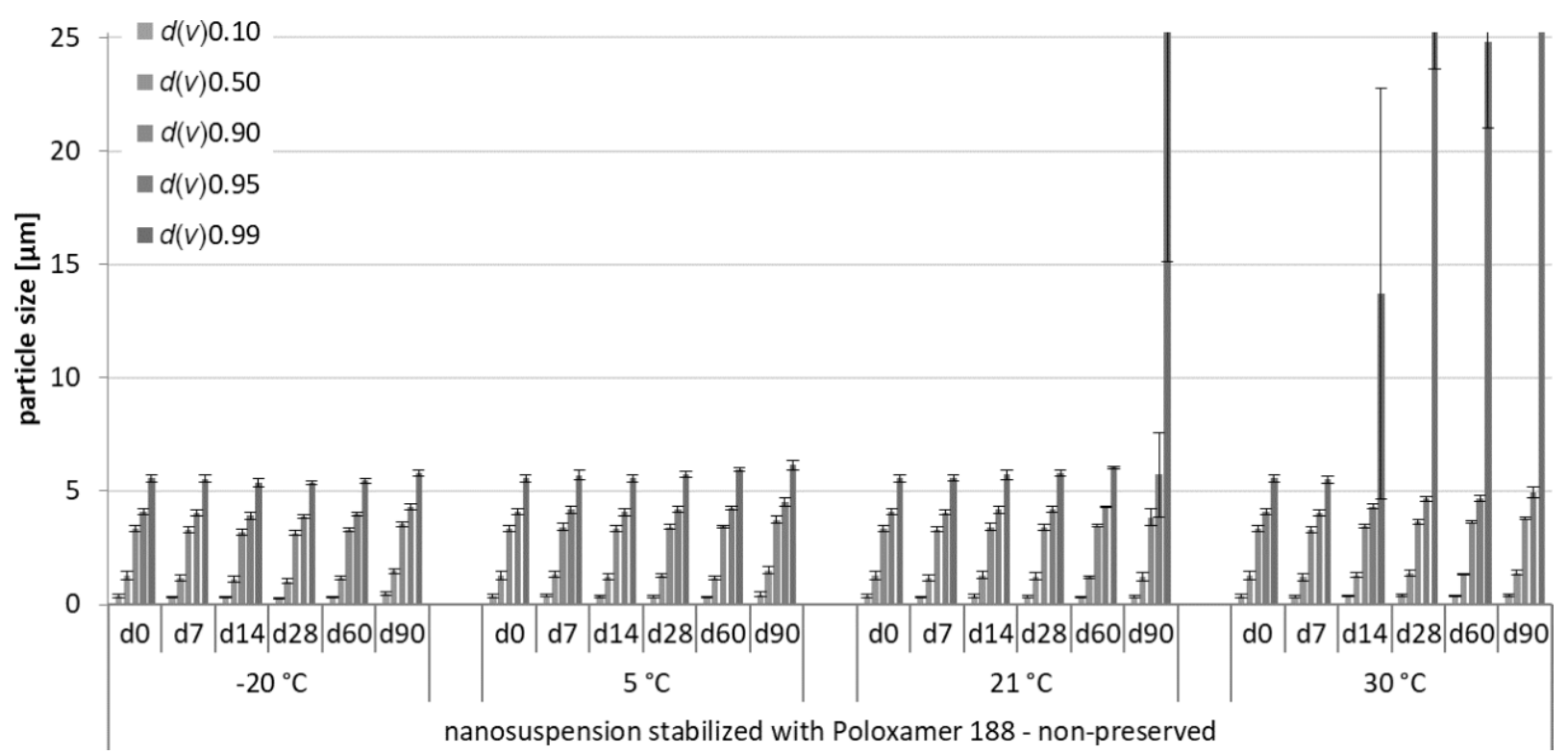

Figure 2: Physical stability during three months of storage at different storage temperatures for the non-preserved nanosuspensions stabilized with Poloxamer 188 (LD data).

ture might change the interaction between preservative and particles and thus the stability. More research is needed to understand these phenomena in detail.
Most interesting results were obtained for the samples that were stored in frozen state. Physically stable formulations, i.e., without pronounced changes in $z$-average, PdI and LD values, were 


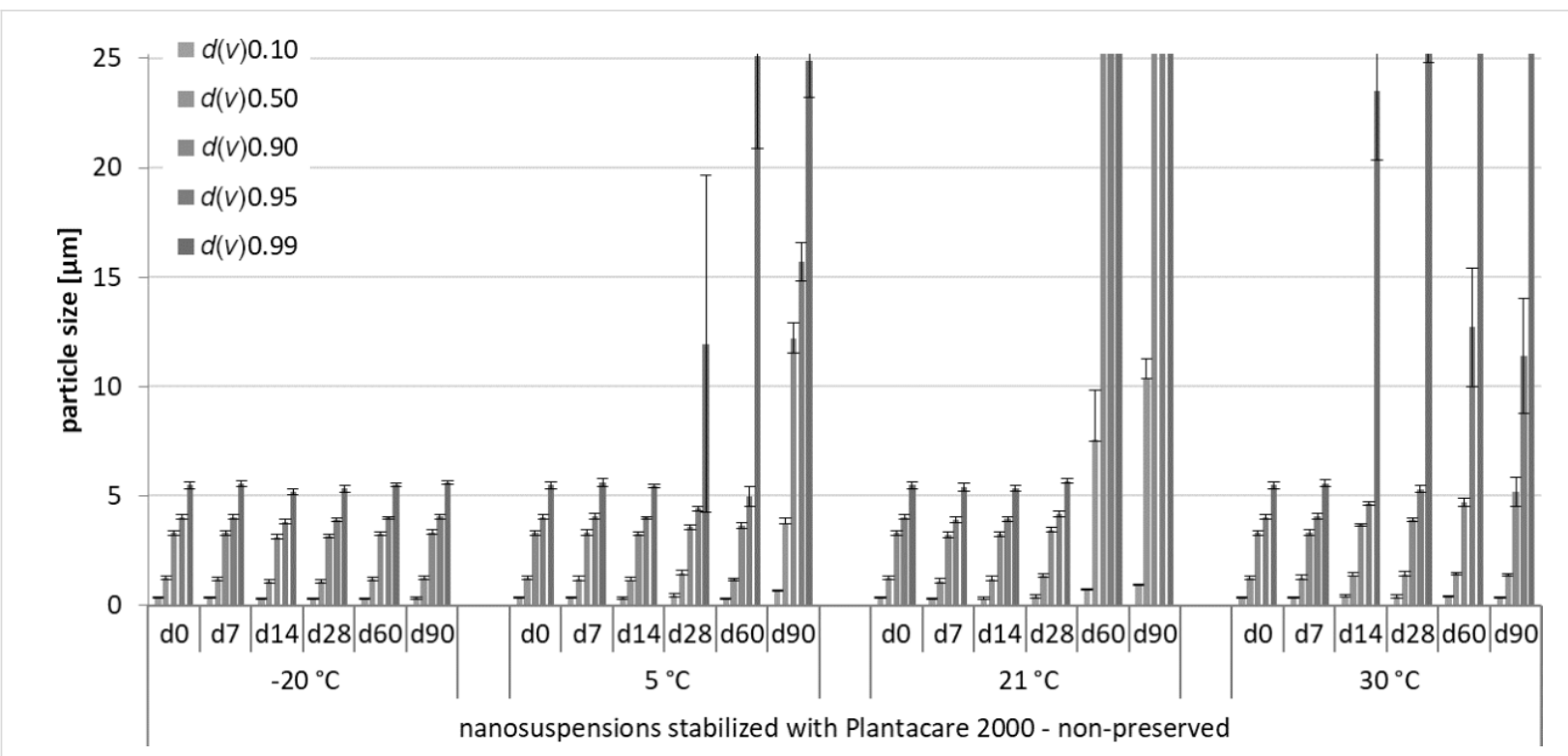

Figure 3: Physical stability during three months of storage at different storage temperatures for the non-preserved nanosuspensions stabilized with Plantacare 2000 (LD data).

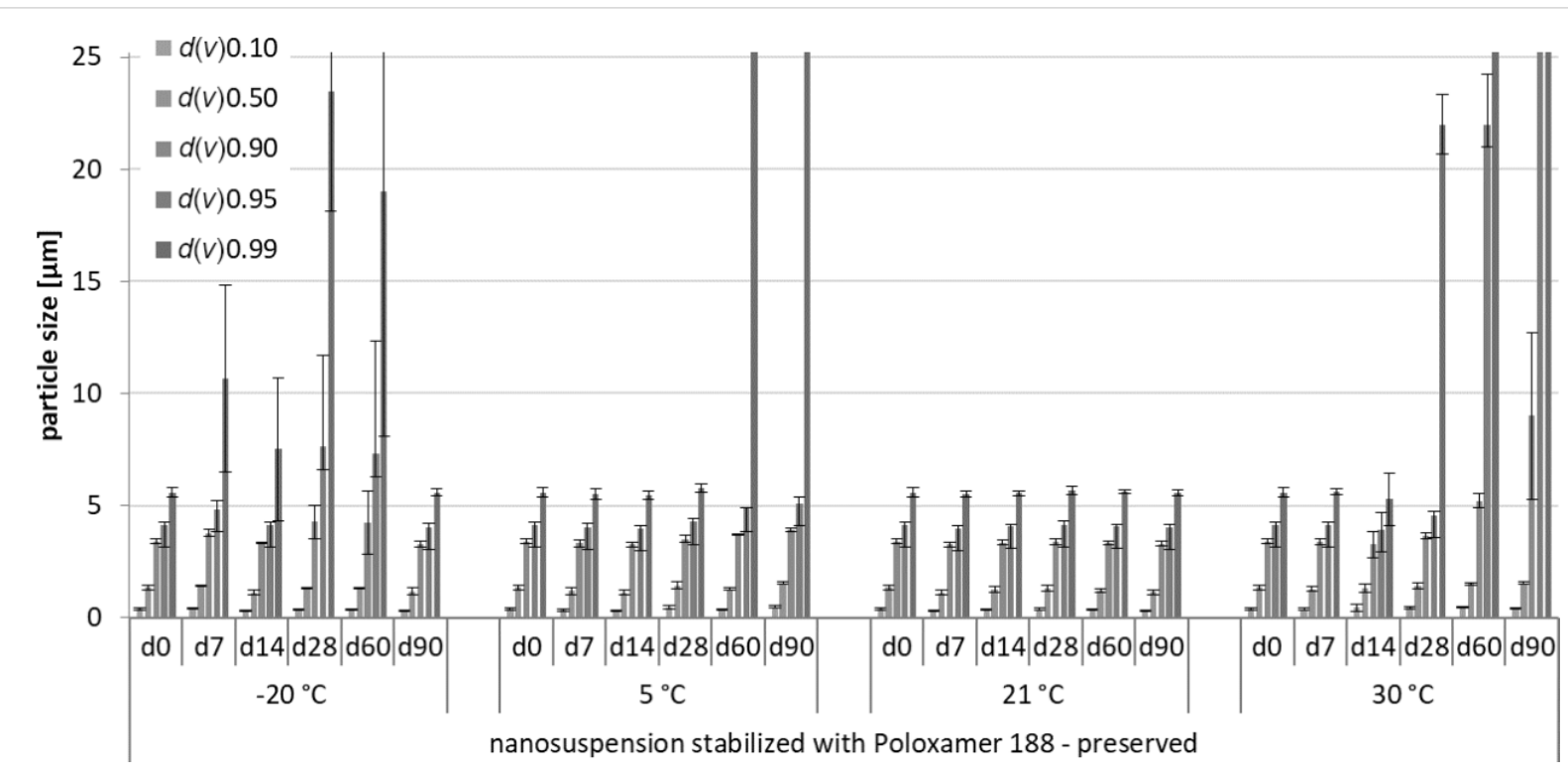

Figure 4: Physical stability during three months of storage at different storage temperatures for the preserved nanosuspensions stabilized with Poloxamer 188 (LD data).

obtained for the non-preserved suspensions when stored at $-20{ }^{\circ} \mathrm{C}$. These results were not expected, because in general it is assumed that freezing of colloidal formulations leads to changes in the stabilizing layer and the particle interactions, being the cause for agglomeration of the nanoparticles. Especially freezing of nanosuspensions that contain dissolved active ingredients in "supersaturation" is hazardous, because due to the reduction in temperature, re-crystallization of dissolved active ingredients can easily occur. Nevertheless, the data obtained from this study indicate that it is possible to freeze-thaw nanosuspensions with good steric and/or electrostatic stabilization, without impairing their physical stability. The later fact seems to be highly important, especially when looking at the data obtained for the frozen and preserved nanosuspensions. Here, it was found that preserved nanosuspensions that were stabilized with PLX 188 became unstable during the freeze-thaw process, whereas Plantacare-stabilized formulations remained stable. 


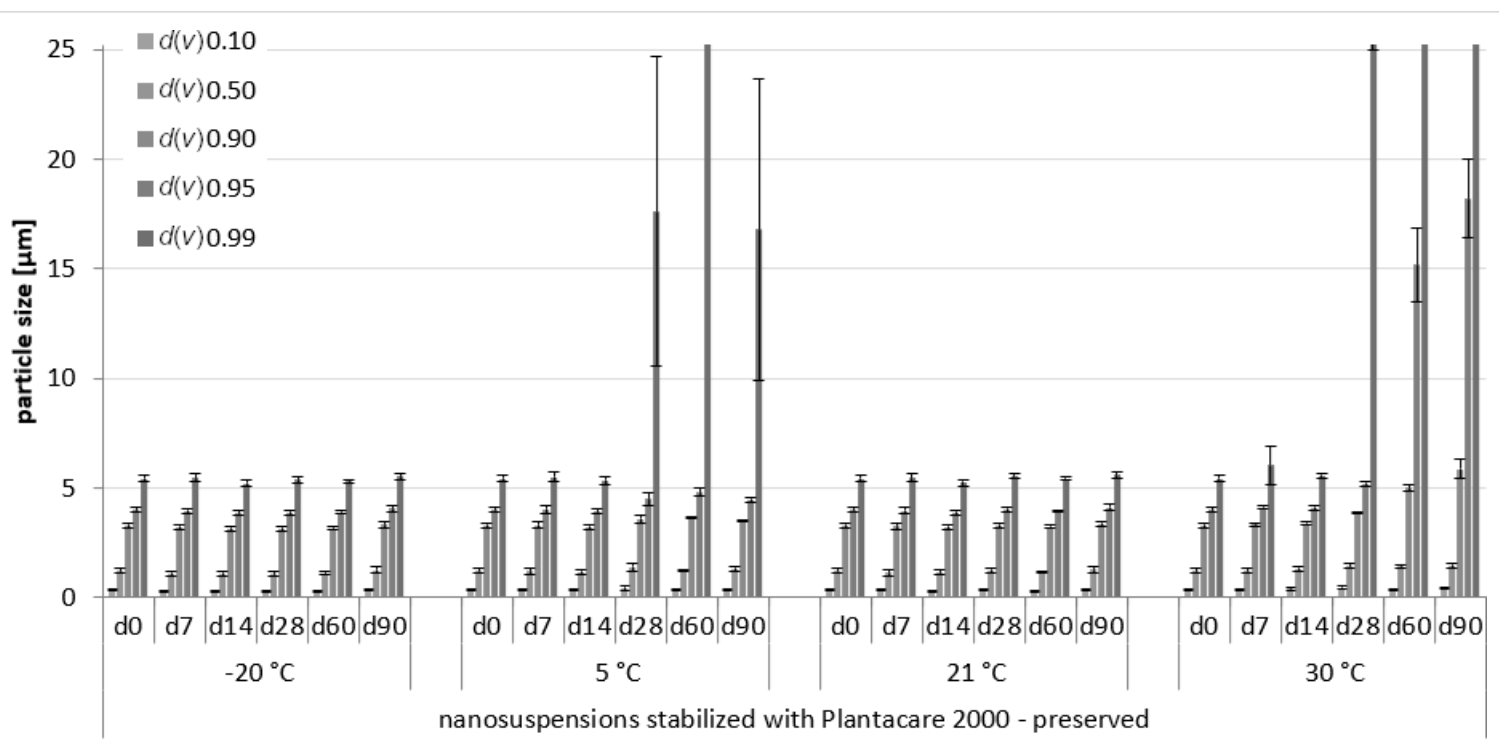

Figure 5: Physical stability during three months of storage at different storage temperatures for the preserved nanosuspensions stabilized with Plantacare 2000 (LD data).

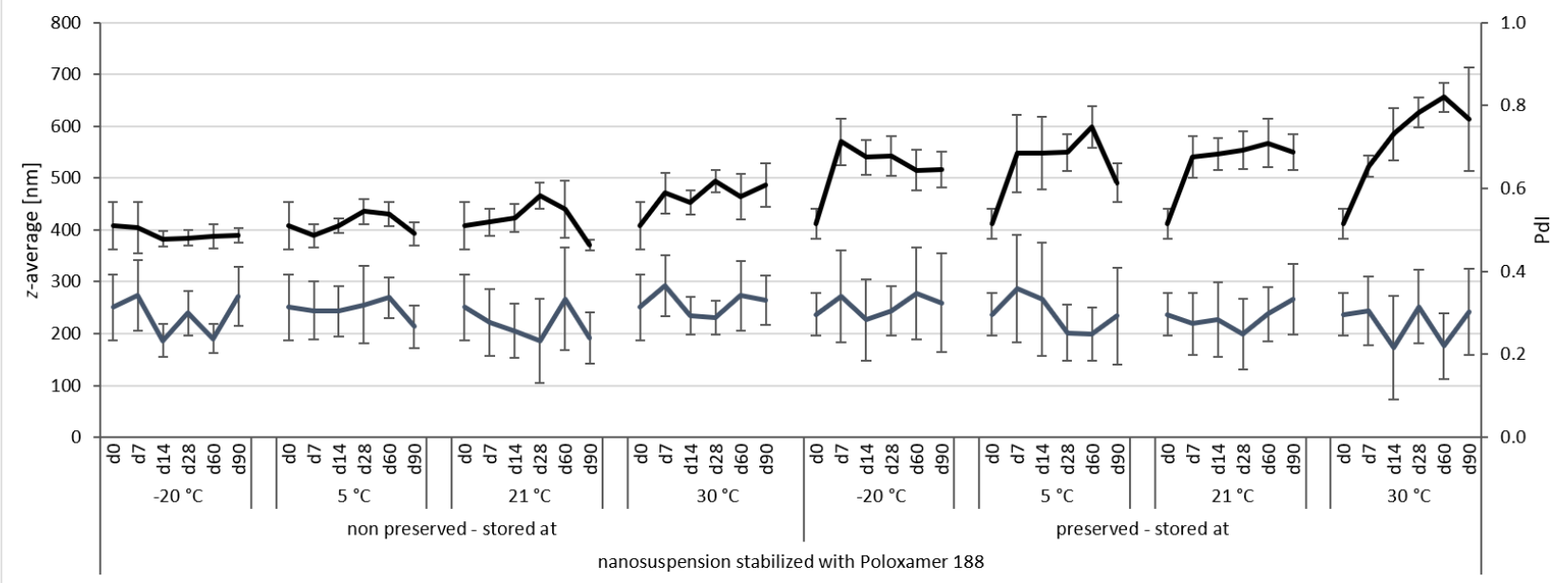

- - -average $[\mathrm{nm}] \longrightarrow$ PdI

Figure 6: Physical stability during three months of storage at different storage temperatures for the nanosuspensions stabilized with Poloxamer 188 (DLS data).

Reasons for the differences might be the different stabilizers that interact differently with the preservative. Poloxamer 188 is a non-ionic surfactant, providing steric stabilization for the nanocrystals. In general, a thick surfactant layer that is indicated by a zeta potential (ZP) near zero should be obtained for good steric stabilization [21]. However, in our study ZP analysis revealed that the thickness of the layer is relatively low (ZP > $20 \mathrm{mV}$, cf. Table 1). Hence, steric stabilization of the formulation stabilized with PLX 188 is relatively poor. Upon the addition of the preservative to the formulations stabilized with PLX 188 a very limited decrease in ZP was detected when the suspensions were analysed in original dispersion medium (c.f. Table 1). This might indicate that small amounts of the non-charged preservative are adsorbed onto the surface of the nanocrystals where it might interact with the polymer layer. This interaction might cause a re-arrangement of the already thin stabilizing layer around the nanocrystals and might therefore explain the decreased stabilization efficacy of the poloxamer in the preserved formulations. The destabilizing effect of PLX 188 in combination with other excipients in nanocrystal formulations was also shown by a study of Beirowski and co-workers, who showed that some combinations of poloxamer and cryoprotectant were unsuitable for stabilizing nanocrystals during a freezing process [22]. 


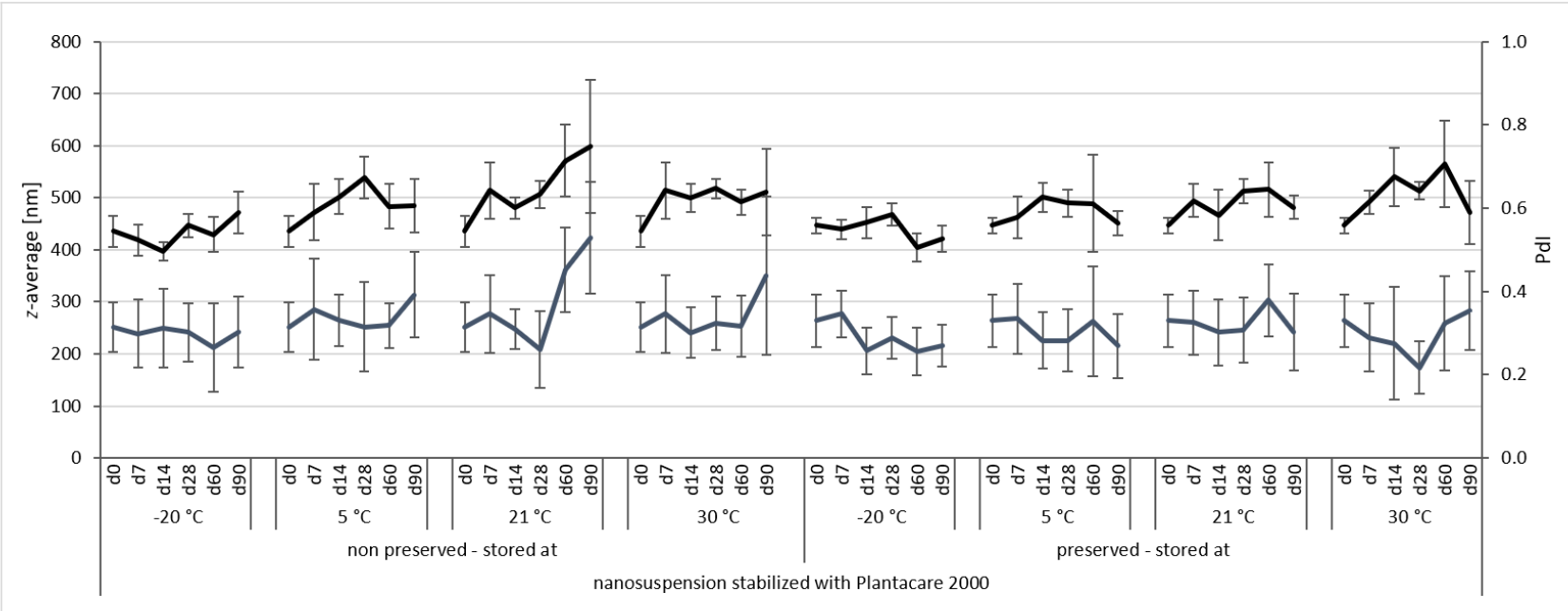

Figure 7: Physical stability during three months of storage at different storage temperatures for the nanosuspensions stabilized with Plantacare 2000 (DLS data).

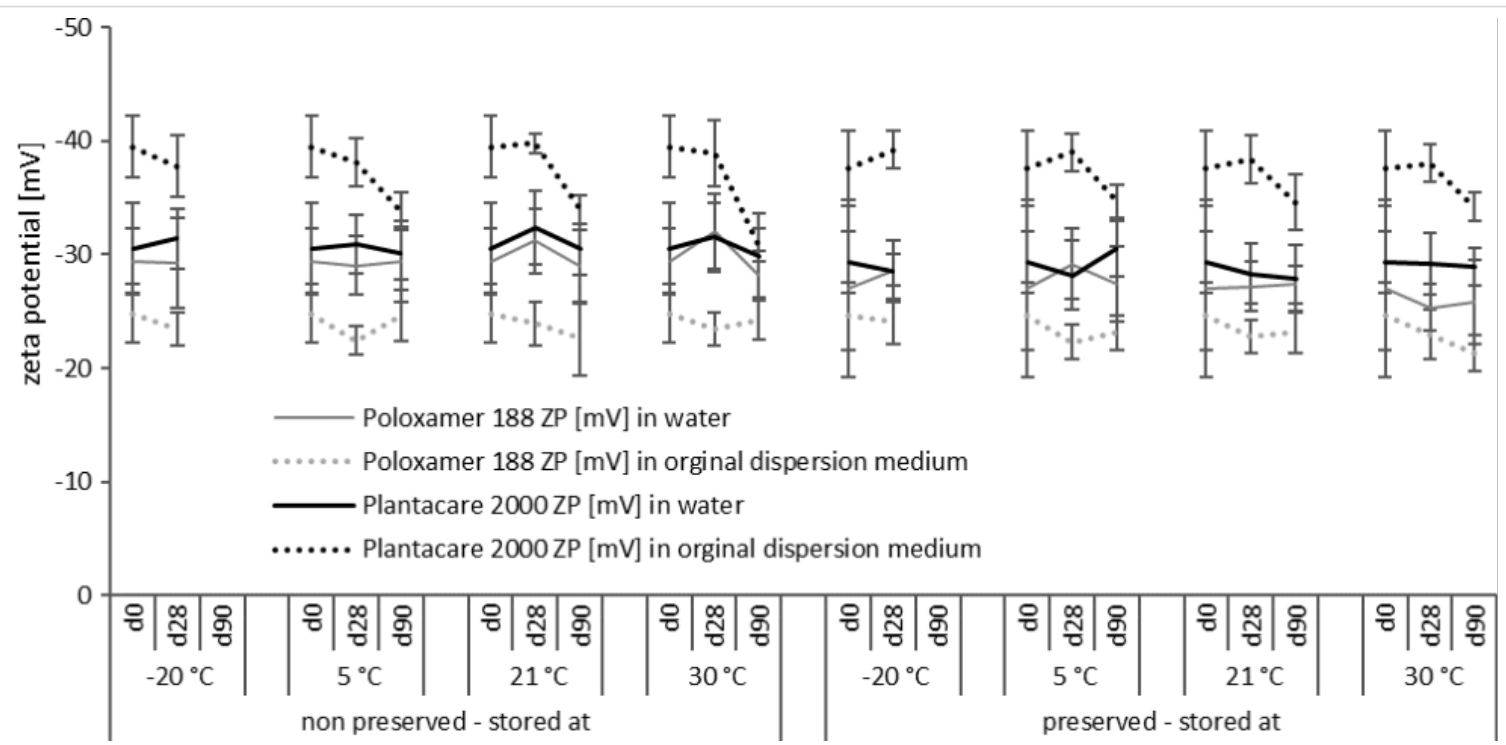

Figure 8: Determination of zeta potentials of the non-preserved and preserved rutin nanosuspensions during three months of storage at different storage temperatures.

In contrast to PLX 188, Plantacare, which is an alkyl polyglucoside, is mostly providing electrostatic stabilization. This can be seen by the differences in ZP analysed in water and original dispersion medium, respectively. The zeta potential is about $-40 \mathrm{mV}$ in original dispersion medium and is reduced to about $-30 \mathrm{mV}$ when analysed in water, because upon dilution with water surfactant is "washed off" from the particle surface, which results in less electrostatic stabilization of the particles. However, due to its chemical structure, Plantacare is also able to provide steric stabilization. This leads to an excellent stabilization capacity combining steric and electrostatic stabilization $[23,24]$. Upon addition of the preservative only very small differences in ZP values were detected for both, water and original dispersion medium (c.f. Table 1), indicating only very minor impairment of the preservative. In fact, Plantacare provides a very efficient stabilization mechanism, which is not significantly impaired by the addition of the preservative. This explains the slightly better physical stability than that of the preserved PLX-stabilized nanosuspensions.

\section{Antioxidant capacity}

The antioxidant capacity (AOC) was measured with the DPPH assay in which the $\mathrm{IC}_{50}$ value is determined. The $\mathrm{IC}_{50}$ value determines the amount of antioxidant needed to scavenge $50 \%$ of 
the free radical. Consequently, the smaller the $\mathrm{IC}_{50}$ value the higher is the antioxidant capacity. In this study, the $\mathrm{IC}_{50}$ values for the different formulations did not change during storage, independent on preservative, storage time and storage temperature (Figure 9). Hence, all these parameters did not affect the AOC of the formulations. As the AOC is an indirect measure for the chemical stability, data indicate excellent chemical stability of all aliquots during storage. The data are in good agreement with a recent study by Müller et al. in which the authors could prove chemical stability of a rutin nanosuspension for more than nine years [25].

\section{Microbial quality}

The growth of bacteria and fungi was determined for all formulations after one, two and three months of storage. All preserved formulations showed excellent microbial quality. No fungi or bacteria were detected during the three months of storage (Figure 10). For the non-preserved nanosuspensions, data indicated that for all formulations the number of bacteria was fairly low upon the production with high-pressure homogenization, which is a well-described technique to reduce the number of bacteria in liquids [26]. The growth of microorganisms during storage was temperature-dependent and was also found to be slightly influenced by the type of stabilizer, i.e., a slightly lower and slower increase in microbial growth was found for the Plantacare-stabilized formulations (Figure 10 and Table 3). A possible reason for this observation could be the antimicrobial activity of the stabilizer Plantacare, which was already described in previous works by Jurado and co-workers [27,28].

Finally, it was found that for all formulations that were stored in frozen state at $-20{ }^{\circ} \mathrm{C}$ no bacterial growth occurred. Hence, the

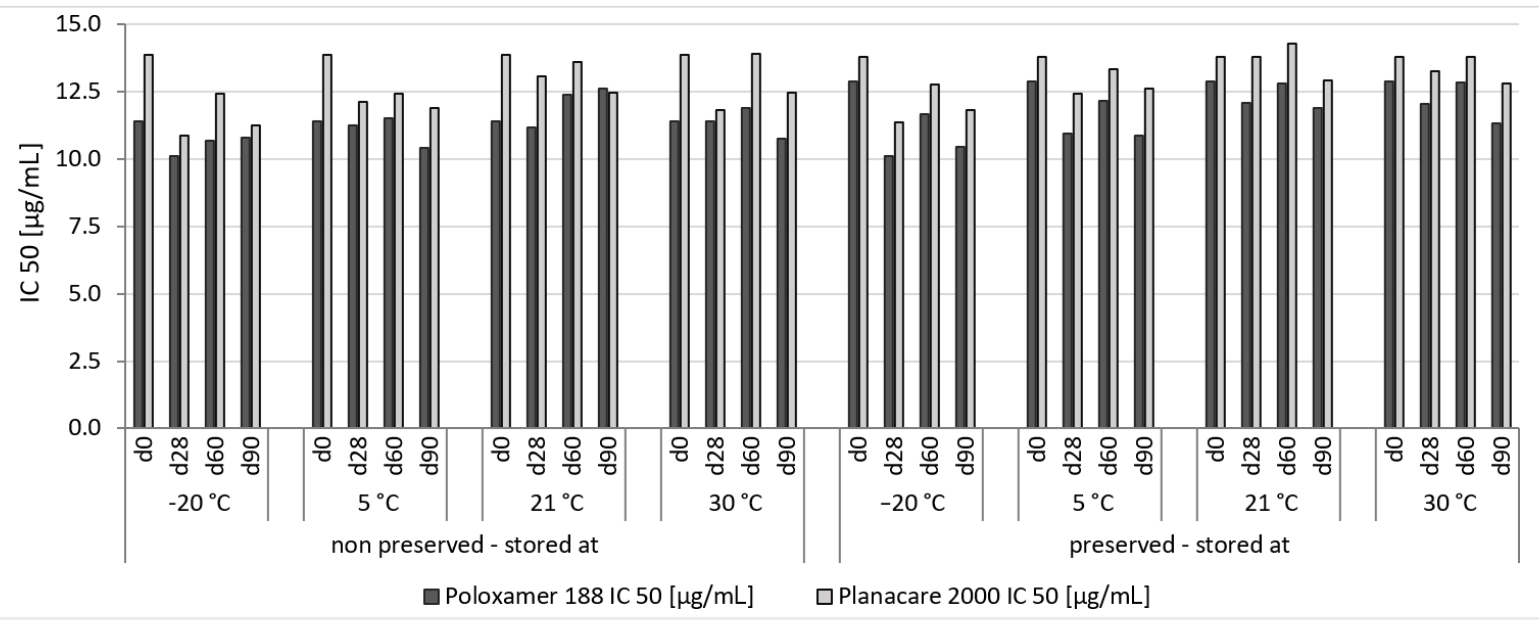

Figure 9: Antioxidant capacity, determined as $\mathrm{IC}_{50}$ value, of the rutin nanosuspensions during storage. No changes in $\mathrm{IC}_{50}$ values were determined during three months of storage, thus indicating good chemical stability of all rutin nanosuspensions.

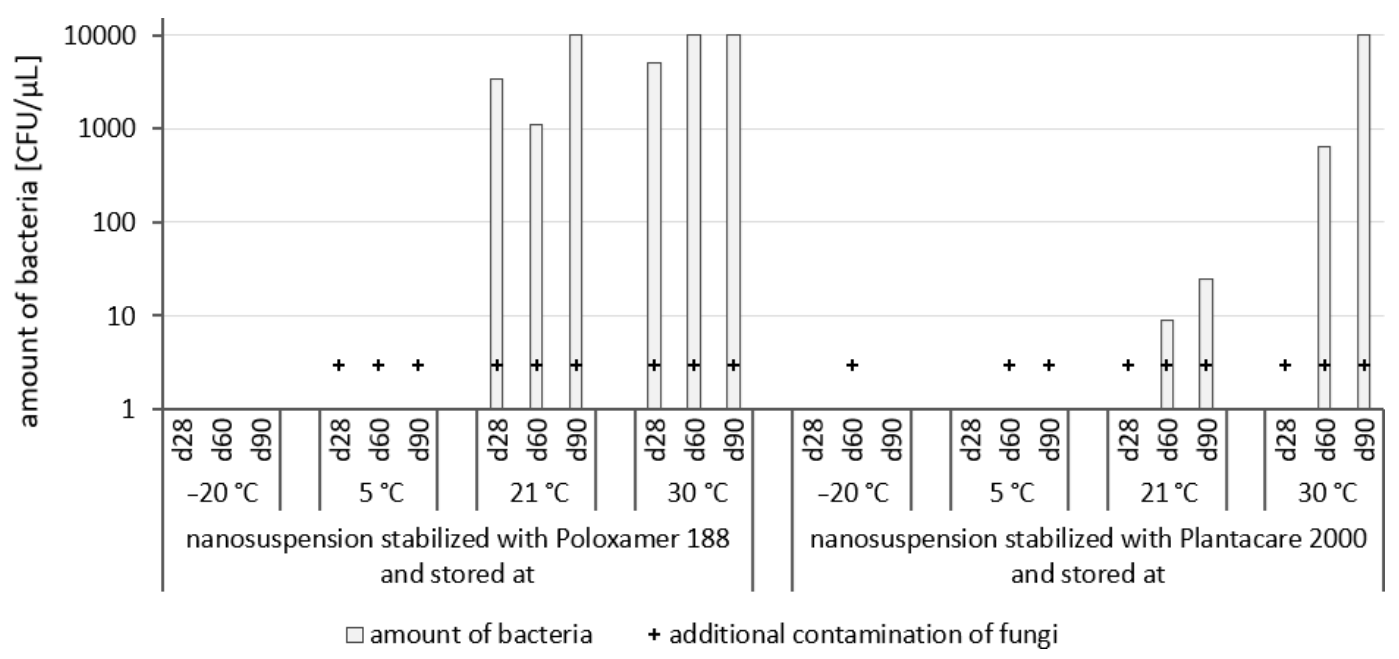

Figure 10: Determination of microbial activity (CFU) during three months of storage. 
Table 3: Determination of microbial quality during three months of storage. P188: rutin nanosuspension stabilized with Poloxamer 188, PLC: rutin nanosuspension stabilized with Plantacare 2000, (+) = preserved with Euxyl 9010, (-) non-preserved.
storage temperature

$-20{ }^{\circ} \mathrm{C}$

$5^{\circ} \mathrm{C}$

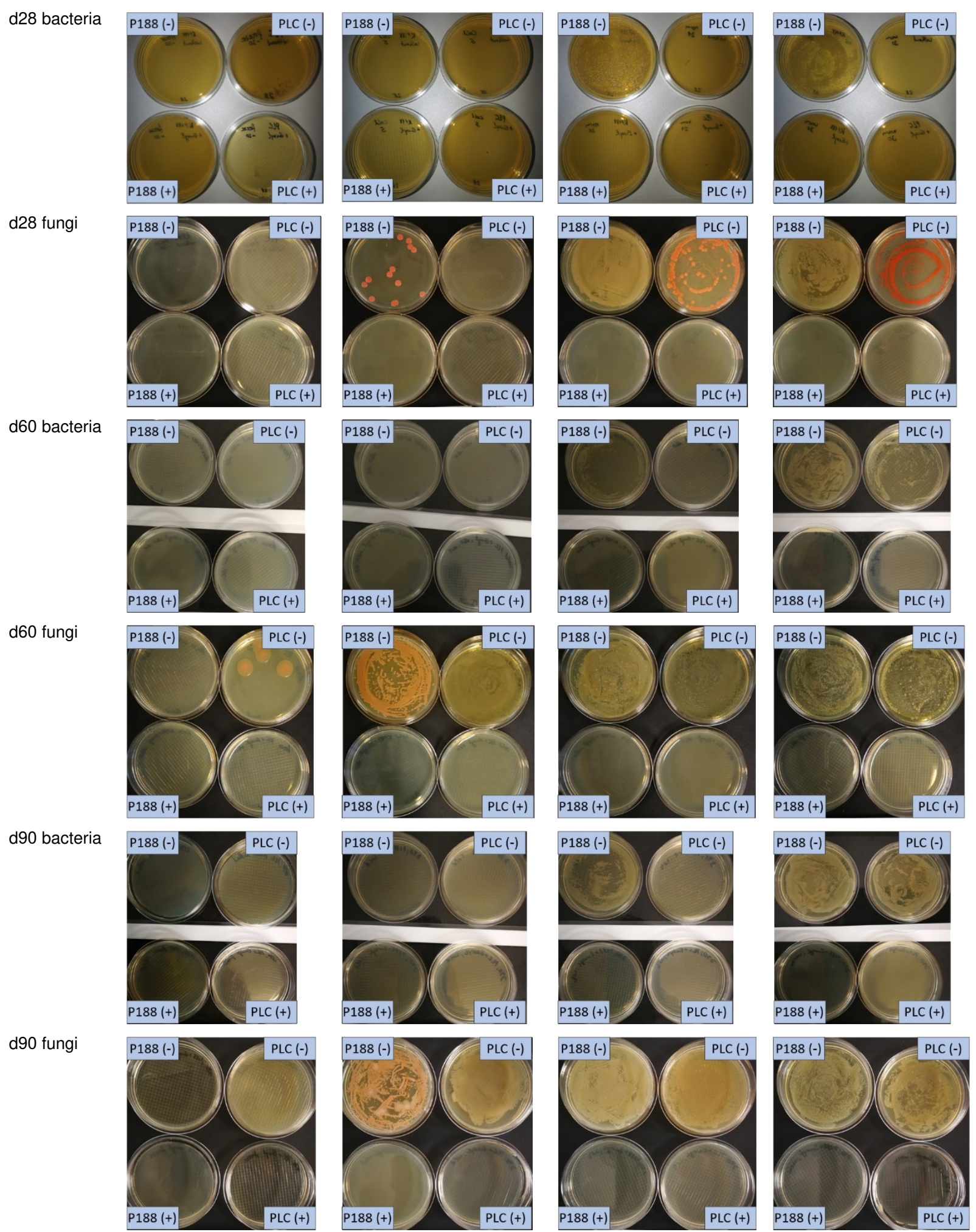


hypothesis, that storing nanosuspensions after production in frozen state might prevent bacterial growth during storage without the use of preservatives, could be confirmed by this set of data. The same trend was also observed for the growth of fungi. However, there was one exception, which was observed for the Plantacare-stabilized formulation at day 60 of storage. At this time point a slight contamination with fungi was observed for one aliquot (Figure 10 and Table 3). However, no contamination with fungi was observed at the next time point, i.e., after 90 days of storage. In fact, after three months of storage at $-20{ }^{\circ} \mathrm{C}$ and upon thawing all non-preserved nanosuspensions were found to possess an excellent microbial quality, as no fungal and no bacterial growth was detected.

These findings so far are very promising and could enable a new concept to produce preservative-free nanosuspensions that can be stored over a longer period until further use or processing into final dosage forms. Preservative-free aqueous nanosuspensions would be a convenient formulation principle, because there will be no need to take possible interactions with preservatives and/or other excipients into consideration. Allergies of consumers and/or regulatory hurdles can also be circumvented with this concept.

The freeze-thaw concept is simple and can be exploited not only in industry but also in early drug development, where nanosuspensions are often used for early formulation of poorly soluble drug candidates. In this environment, the freeze-thaw concept could improve the predictability of screenings. At present, due to the lack of microbial stability, nanosuspensions need to be prepared shortly before the experiments, i.e., assays, cell culture or in vivo studies, are performed. Any repeating of the tests or continued tests will require the production of new suspensions, which might possess slightly different properties, which in turn might then cause differences in the (in vivo) data. By using thawed nanosuspensions from only one batch, these variations could be circumvented.

In conclusion, the freeze-thaw concept was shown to be a simple method to prevent microbial contamination during storage of aqueous rutin nanosuspensions. The new method is believed to enable new possibilities for the use of nanosuspensions and thus can be seen as a highly promising concept, not only in pharma, but also in food and cosmetics. Next steps should now investigate if the concept can also be exploited for other active ingredients and other stabilizers.

\section{Conclusion}

Preserved and non-preserved rutin nanosuspensions stabilized with different stabilizers were produced in this study and were stored for three months at different storage temperatures.
During this time physical stability and microbial quality were monitored. In addition, the antioxidant capacity, as an indicator for the chemical stability, was assessed. All formulations were chemically stable over the whole time of observation. Physical stability was influenced by the type of surfactant, the preservative and the storage temperature. Preserved samples were only stable when stored at room temperature. Storage at higher or lower temperatures strongly impaired their physical stability. However, the microbial quality was excellent for all preserved nanosuspensions. Non-preserved samples possessed a better physical stability than the preserved nanosuspension. Proving again that preservatives impair the physical stability of nanosuspensions. Most interestingly, it was found that freezing did not alter the physical stability of the non-preserved suspensions. Hence, nanosuspensions could be frozen, stored up to three months at $-20{ }^{\circ} \mathrm{C}$ and possessed unchanged particle sizes upon thawing. Storage at $-20{ }^{\circ} \mathrm{C}$ also prevented bacterial growth of the non-preserved nanosuspensions, whereas storage at higher temperatures caused microbial contamination of the suspensions. The freeze-thaw concept was therefore found to be a suitable method to produce not only physically and chemically but also microbially stable rutin nanosuspensions. More research is now needed to investigate if the method can also be transferred to other nanosuspensions or nanosized formulations. All in all, the method seems to be a promising method to enable long time storage of aqueous nanosuspensions with excellent stability and without the use of preservatives. It can be used for improved formulation development of poorly soluble active ingredients in both lab scale and industrial scale.

\section{Experimental \\ Materials}

Rutin was purchased from Denk Ingredients GmbH (Germany). The stabilizers Poloxamer 188 (PLX 188, Kolliphor ${ }^{\circledR}$ P 188) and alkyl polyglucoside C8-C16 (Plantacare ${ }^{\circledR} 2000$ UP) were kindly provided from BASF AG (Germany). The preservative was composed of $90 \%(\mathrm{w} / \mathrm{w}) 2$-phenoxyethanol and $10 \%(\mathrm{w} / \mathrm{w})$ of 1,2-propanediol as ready to use mixture (Euxyl $\left.{ }^{\circledR} 9010\right)$ and was obtained from Schülke \& Mayr GmbH (Germany). Purified water was obtained from a PURELAB Flex 2 (ELGA LabWater \& Veolia, Germany). All other analytical chemicals were of analytical grade and used as received.

\section{Methods}

\section{Production of nanosuspensions}

Rutin nanosuspensions [16-19] were produced by high-pressure homogenization (HPH) using an EmulsiFlex-C50 (Avestin, Germany). For this, bulk suspensions containing 5\% (w/w) rutin and $1 \%(\mathrm{w} / \mathrm{w})$ surfactant were prepared. The pre-dispersions were homogenized with a high-speed stirrer (D-27, Miccra GmbH, Germany) at 24,000 rpm for $5 \mathrm{~min}$ in continu- 
ous mode and were subsequently subjected to HPH (20 cycles at 1500 bar). During homogenization and between each cycle the suspensions were cooled to below $10^{\circ} \mathrm{C}$ by using a cooling bath to avoid heating of the suspensions and subsequent agglomeration of the crystals [29].

\section{Characterization of nanosuspensions}

Determination of particle size and physical stability: Nanosuspensions were characterized regarding size by three different and independent methods. The hydrodynamic diameter and the polydispersity ( $z$-average ( $z$-ave) and PdI) were analysed by dynamic light scattering (DLS) with a Zetasizer Nano ZS (Malvern Panalytical GmbH, Germany). As DLS measurements, when used as stand-alone method for the characterization of submicron-sized particles, can be misleading because larger sized particles are not detected [30-33], light microscopy (Olympus BX53, equipped with an Olympus SC50 CMOS color camera, Japan) and laser diffraction (LD) were used as additional techniques to securely detect possible larger particles and agglomerates within the suspensions. By LD analysis the volumetric median diameters $d(v) 0.1, d(v) 0.5, d(v) 0.9$, $d(v) 0.95$ and $d(v) 0.99$ were analysed with a Mastersizer 3000 (Malvern Panalytical GmbH, Germany). Particle diameters were calculated with Mie-theory by using 1.57 as real refractive index and 0.01 as imaginary refractive index.

The zeta potential (ZP) of the nanocrystals was determined in water (adjusted to a constant conductivity of $50 \mu \mathrm{S} / \mathrm{cm}$ ) and in original dispersion media, i.e., surfactant solution, containing either 1\% (w/w) PLX 188 or Plantacare 2000, respectively. Measurements were performed via laser-Doppler-anemometry (LDA) by using a Zetasizer Nano ZS (Malvern Panalytical $\mathrm{GmbH}$, Germany), which determines the electrophoretic mobility (EM), which was then converted into the ZP by using the Helmholtz-Smoluchowski equation [21].

Determination of antioxidant capacity: The antioxidant capacity was assessed by calculating the $\mathrm{IC}_{50}$ value, which was determined by using the DPPH assay [34]. DPPH (1,1diphenyl-2-picryl-hydrazyl, Sigma-Aldrich, Germany) is a free radical that can be reduced by antioxidants. Upon reduction the colour of the free radical changes and thus the amount of reduced DPPH can be accessed via UV-vis spectroscopy. For the determination of the $\mathrm{IC}_{50}$ values, $100 \mu \mathrm{L}$ of the samples containing different concentrations of the nanocrystals (200, $100,50,25,12.5,6.25,3.125 \mu \mathrm{g} / \mathrm{mL}$ ) were added to $100 \mu \mathrm{L}$ of a $0.3 \mathrm{mM}$ methanolic solution of DPPH. After 30 min incubation time in the dark, the absorbance was measured by a UV-vis plate reader (Multiskan GO, Thermo scientific, Germany) at $517 \mathrm{~nm}$. The inhibition activity (inhibition [\%]) was calculated as

$$
\text { inhibition }[\%]=\left(1-A_{\text {sample }} / A_{0}\right) \cdot 100 \text {, }
$$

where $A_{\text {sample }}$ is the absorbance of the sample and $A_{0}$ is the absorbance of the control (DPPH solution). The resulting linear function of inhibition against concentration was used to calculate the $\mathrm{IC}_{50}$ value $(\mu \mathrm{g} / \mathrm{mL})$. The $\mathrm{IC}_{50}$ value represents the concentration needed to scavenge $50 \%$ of the free radical. Rutin, which was used in this study as model drug, is a well-known antioxidant. Hence, if chemical degradation of the active ingredient occurs, changes in the $\mathrm{IC}_{50}$ value during storage can be observed [35]. As methanol is a good solvent for rutin, the addition of the nanocrystals to the methanolic DPPH solution led to a complete dissolution of the rutin nanocrystals. Hence, all rutin remaining in the formulations was dissolved during the test and thus the DPPH assay was used as a surrogate for the determination of the chemical stability.

Determination of microbial quality: To verify the biological stability, a simple agar plate test after Ph. Eur. 8 was used. The agar (Müller-Hinton agar, Sigma) was dispersed in water, autoclaved and directly poured into sterile petri dishes $(60 \mathrm{~mm}$ in diameter) at 28, 60 and 90 days after preparation of the nanosuspensions and used immediately after cooling. Subsequently, $10 \mu \mathrm{L}$ of the nanosuspension or a 1:100 dilution in water were distributed evenly over the entire surface by means of a cell spreader. For detection of existing bacteria, the agar plate containing the suspension or dilution was incubated for $24 \mathrm{~h}$ at $36{ }^{\circ} \mathrm{C}$ and $90 \%$ humidity. Fungal contamination was detected after 7 days at $25{ }^{\circ} \mathrm{C}$ storage by using undiluted nanosuspensions. For evaluation, the visible bacterial colonies were counted, or the presence of fungal growth was noted. With dense colonization, the number of colony forming units (CFU) was set to $10000 \mathrm{CFU} / \mu \mathrm{L}$.

\section{ORCID ${ }^{\circledR}$ iDs}

Cornelia M. Keck - https://orcid.org/0000-0001-8888-2340

\section{References}

1. Auweter, H.; Bohn, H.; Heger, R.; Horn, D.; Siegel, B.; Siemensmeyer, K. Precipitated water-insoluble colorants in colloid disperse form. U.S. Patent 6,494,924B1, Dec 12, 2002.

2. List, M.; Sucker, H. Pharmaceutical colloidal hydrosols for injection. G.B. Patent 2,200,048, July 27, 1988.

3. Müller, R. H.; Becker, R.; Kruss, B.; Peters, K. Pharmaceutical nanosuspensions for medicament administration as systems with increased saturation solubility and rate of solution. U.S. Patent 5,858,410, Dec 1, 1999.

4. Liversidge, G. G.; Cundy, K. C.; Bishop, J. F.; Czekai, D. A. Surface modified drug nanoparticles. U.S. Patent 5,145,684, Sept 8, 1992.

5. Wu, W.; Nancollas, G. H. J. Solution Chem. 1998, 27, 521-531. doi:10.1023/a:1022678505433 
6. Müller, R. H.; Gohla, S.; Keck, C. M. Eur. J. Pharm. Biopharm. 2011, 78, 1-9. doi:10.1016/j.ejpb.2011.01.007

7. Müller, R. H.; Keck, C. M. Int. J. Pharm. 2010, 390, 1-2. doi:10.1016/j.ijpharm.2010.02.005

8. Fan, M.; Geng, S.; Liu, Y.; Wang, J.; Wang, Y.; Zhong, J.; Yan, Z.; Yu, L. Curr. Pharm. Des. 2018, 24, 2416-2424. doi:10.2174/1381612824666180515154109

9. Patel, V.; Sharma, O. P.; Mehta, T. Expert Opin. Drug Delivery 2018, 15, 351-368. doi:10.1080/17425247.2018.1444025

10. Sharma, O. P.; Patel, V.; Mehta, T. Drug Delivery Transl. Res. 2016, 6 , 399-413. doi:10.1007/s13346-016-0292-0

11. Keck, C. M.; Müller, R. H. Eur. J. Pharm. Biopharm. 2006, 62, 3-16. doi:10.1016/j.ejpb.2005.05.009

12. Agarwal, V.; Bajpai, M. Recent Pat. Nanotechnol. 2015, 9, 178-194. doi:10.2174/1872210510999151126112644

13. Al Shaal, L.; Mishra, P. R.; Müller, R. H.; Keck, C. M. Pharmazie 2014, 69, 173-182. doi:10.1691/ph.2014.3032

14. Kobierski, S.; Ofori-Kwakye, K.; Müller, R. H.; Keck, C. M. Pharmazie 2011, 66, 942-947. doi:10.1691/ph.2011.1038

15. Obeidat, W. M.; Schwabe, K.; Müller, R. H.; Keck, C. M. Eur. J. Pharm. Biopharm. 2010, 76, 56-67. doi:10.1016/j.ejpb.2010.05.001

16. Mauludin, R.; Müller, R. H.; Keck, C. M. Int. J. Pharm. 2009, 370 , 202-209. doi:10.1016/j.jpharm.2008.11.029

17. Mauludin, R.; Müller, R. H.; Keck, C. M. Eur. J. Pharm. Sci. 2009, 36, 502-510. doi:10.1016/j.ejps.2008.12.002

18. Scholz, P.; Arntjen, A.; Müller, R. H.; Keck, C. M. Int. J. Pharm. 2014, 465, 388-395. doi:10.1016/j.jpharm.2014.02.026

19. Scholz, P.; Keck, C. M. Int. J. Pharm. 2015, 482, 27-37. doi:10.1016/j.ijpharm.2014.11.008

20. Al Shaal, L.; Müller, R. H.; Keck, C. M. Pharmazie 2010, 65, 86-92.

21. Müller, R. H. Zetapotential und Partikeladung in der Laborpraxis; Wissenschaftliche Verlagsgesellschaft: Suttgart, Germany, 1996.

22. Beirowski, J.; Inghelbrecht, S.; Arien, A.; Gieseler, H. J. Pharm. Sci. 2011, 100, 1958-1968. doi:10.1002/jps.22425

23. Kobierski, S.; Ofori-Kwakye, K.; Müller, R. H.; Keck, C. M. Pharmazie 2009, 64, 741-747. doi:10.1691/ph.2009.9097

24. Kovacevic, A.; Savic, S.; Vuleta, G.; Müller, R. H.; Keck, C. M. Int. J. Pharm. 2011, 406, 163-172. doi:10.1016/j.ijpharm.2010.12.036

25. Müller, R. H.; Hespeler, D.; Keck, C. M. Euro Cosmetics 2018, 11, 6-9.

26. Diels, A. M. J.; Michiels, C. W. Crit. Rev. Microbiol. 2006, 32, 201-216. doi:10.1080/10408410601023516

27. Jurado, E.; Fernández-Serrano, M.; Núñez Olea, J.; Lechuga, M.; Jiménez, J. L.; Ríos, F. Bull. Environ. Contam. Toxicol. 2012, 88, 290-295. doi:10.1007/s00128-011-0479-5

28. Jurado, E.; Fernández-Serrano, M.; Núñez-Olea, J.; Luzón, G.; Lechuga, M. Water Sci. Technol. 2009, 59, 2351-2358. doi:10.2166/wst.2009.266

29. Keck, C. M. Cyclosporine nanosuspensions: Optimised size characterisation \& oral formulations. Ph.D. Thesis, Freie Universität Berlin, Berlin, Germany, 2006.

30. Gioria, S.; Caputo, F.; Urbán, P.; Maguire, C. M.; Bremer-Hoffmann, S.; Prina-Mello, A.; Calzolai, L.; Mehn, D. Nanomedicine (London, U. K.) 2018, 13, 539-554. doi:10.2217/nnm-2017-0338

31. Knoth, D.; Keck, C. M. Phys. Status Solidi A 2018, 215, 1700962. doi:10.1002/pssa.201700962

32. Keck, C. M.; Müller, R. H. Int. J. Pharm. 2008, 355, 150-163. doi:10.1016/j.ijpharm.2007.12.004

33. Keck, C. M. Int. J. Pharm. 2010, 390, 3-12. doi:10.1016/j.ijpharm.2009.08.042
34. Kedare, S. B.; Singh, R. P. J. Food Sci. Technol. 2011, 48, 412-422. doi:10.1007/s13197-011-0251-1

35. Klimczak, I.; Małecka, M.; Szlachta, M.; Gliszczyńska-Świgło, A. J. Food Compos. Anal. 2007, 20, 313-322. doi:10.1016/j.jfca.2006.02.012

\section{License and Terms}

This is an Open Access article under the terms of the Creative Commons Attribution License (http://creativecommons.org/licenses/by/4.0). Please note that the reuse, redistribution and reproduction in particular requires that the authors and source are credited.

The license is subject to the Beilstein Journal of Nanotechnology terms and conditions:

(https://www.beilstein-journals.org/bjnano)

The definitive version of this article is the electronic one which can be found at: doi:10.3762/bjnano.10.185 